\title{
Luciana TRIMANO*
}

Radio y escuela en el proceso de construcción del patrimonio natural en la comunidad rural del valle de Traslasierra

The school and radio in construction process of natural heritage at the rural community in the Valley of Traslasierra

\section{Paulina-Beatriz EMANUELLI*}

\section{Resumen}

Las comunidades de Traslasierra, en el oeste de la provincia de Córdoba, sufren cambios profundos en el uso de la tierra. Áreas rurales que mantenían una valiosa agrodiversidad, como ganadería, cultivos anuales, hortalizas, frutales, e importantes áreas del ecosistema nativo, progresivamente se transformaron debido al avance de la agricultura industrial, la minería y los desarrollistas inmobiliarios. Como dato característico de la realidad de la zona surge: la emigración de mano de obra, la pérdida de productores y saberes ancestrales sobre producciones autóctonas, la falta de oportunidades de capacitación, y un escaso desarrollo de acciones asociativas. En este escenario, es imprescindible pensar una producción estable dentro del contexto de una organización social que proteja la integridad de los recursos naturales y que

\begin{abstract}
${ }^{1}$
The communities in Traslasierra, in the west of Córdoba province, undergo deep changes in the use of the land. Rural areas which used to maintain a valuable agrobiodiversity like cattle raising, annual crops, vegetables, fruit trees and important areas of the native ecosystem, were transformed progressively due to the advance of industrial agriculture, the mining industry and estate agents or housing developers. As a real fact of the life in that region, several situations emerge such as the emigration of labor force, the loss of farmers along with the missing ancestral wisdom on native productions, the lack of training opportunities and a poor development of associative actions. Given this situation, it is essential to think about a steady production within the context of a social organization in which the integrity of natural resources are protected and a
\end{abstract}

1 Traducción realizada por la Profesora Marta Masera. Escuela de Ciencias de la Información. Facultad de Derecho y Ciencias Sociales. Universidad Nacional de Córdoba, Argentina. 
asegure la interacción armónica de los seres humanos, el agroecosistema y el medio ambiente. Así, se piensa el acercamiento de la escuela pública a la radio, en una alianza mutua. Desde el intercambio de saberes, se trabaja con niños y niñas de $4^{\circ}, 5^{\circ}$ y $6^{\circ}$ grado. A través de la práctica radiofónica y la problematización de temáticas ambientales y agroecológicas, ellos construyen la realidad sobre su entorno social, cultural y ambiental; y desde dicho marco aportan al cuidado del hábitat local. Se convierten en promotores y multiplicadores para la defensa y resguardo del medio ambiente.

\section{Palabras clave}

Construcción de la realidad; hábitat; radio participativa.

\section{Sumario}

1. Introducción. 2. La construcción de la realidad socioambiental en el valle de Traslasierra. 3. Traslasierra, distintas perspectivas teóricas. 4. Estrategia metodológica. 5. Los niños y niñas tienen la palabra. 6. Talleres de comunicación y ambiente. 7. Referencias bibliográficas. good and harmonious interaction among human beings, the agroecosystem and the environment can be guaranteed. It is in this way that the state schools get closer to the radio in a mutual alliance, working each other. All the work carried out with children from $4^{\text {th }}, 5^{\text {th }}$ and $6^{\text {th }}$ grade school is considered an opportunity to share knowledge. Through the broadcasting performance and the problematization of environmental and agroecological issues, children can construct a real world based on its environmental, cultural and social setting; and it is from this approach that they can contribute to the care of the local habitat. Therefore, they become promoters for the defense and protection of the environment resulting in a multiplying effect.

\section{Keywords}

Construction of reality; habitat; participatory radio.

\section{Summary}

1. Introduction. 2. The construction of socio environmental reality in valle de Traslasierra. 3. Traslasierra, different theoretical perspectivas. 4. Methodological strategy. 5. Children have their Word. 6. Workshops of communication and environment. 7. References. 
1 El siguiente artículo es el resultado de un proyecto llevado a cabo en el año 2010 en la localidad de Las Calles, Valle de Traslasierra, Córdoba, Argentina. Su título formal es "Recuperando saberes ambientales y agroecológicos desde la práctica radial en la escuela. Caso Las Calles, Traslasierra, Córdoba". La entidad financiadora: Secretaría de Extensión Universitaria. Universidad Nacional de Córdoba. Argentina.

\section{Introducción 1}

Desde hace más de quince años, la agricultura argentina transita un proceso de transformación, que se materializa en el aumento de la producción y la productividad, la extensión de la superficie y el crecimiento de los saldos exportables. Dichos cambios fueron promovidos, a inicios de los años 90, por la implementación del modelo político-económico neoliberal. Situación que posicionó, al sector agropecuario, en el mercado internacional y llevo al conjunto de actividades a ser analizadas en términos de negocio (Grosso y Albaladejo, 2009: 117). El modelo de ruralidad globalizada se fue cristalizando en el medio productivo y en la prensa, a través de la noción de "paradigma de los agronegocios". Este acontecer, llevo al desplazamiento de un tipo de agricultura familiar, ampliamente practicada por los chacareros y los pequeños productores descendientes de las primeras generaciones emigradas de Europa. El modelo sojero es un sistema de producción que va más allá de la mera adopción del cultivo transgénico. La expansión de ese sistema, es el promotor de la división social del trabajo dentro del sector y entre sectores, al tiempo que prioriza las necesidades del consumidor global respecto del local (Hernández, 2009: 39-40).

Todo ello, viene a decir que la modernización no solo se estableció en el marco de lo productivo; sino también en las condiciones de vida de los pobladores. Tal es el caso del abandono de diversas actividades que habían dominado hegemónicamente- la economía rural, por el cultivo masivo de soja. De esta forma, asistimos a "una trasformación socioproductiva y territorial dada por el avance de un modelo de producción agrícola altamente tecnificado y demandante de capital que asemeja a la agricultura a la producción industrial. En tanto, se borran las particularidades locales, (...) se relegan los saberes tradicionales, generándose exclusión y dependencia en medio de la riqueza" (Grosso y Albaladejo, 2009: 121).

En este contexto, el Valle de Traslasierra -al oeste de la provincia de Córdoba (Argentina)-advierte, cómo áreas que mantenían una valiosa agrodiversidad (ganadería, cultivos anuales, hortalizas, frutales, e importantes áreas del ecosistema nativo), progresivamente se transforman por el avance de la agricultura industrial, la minería y los desarrollistas inmobiliarios. En consecuencia, la emigración de mano de obra, la pérdida de productores y sus saberes ancestrales sobre producciones y espacios de comercialización de productos autóctonos, la falta de oportunidades de capacitación y escaso desarrollo de acciones asociativas, se convierten en datos característicos de la realidad de la zona. 
2 Desde esta perspectiva se entienden las prácticas sociales de la comunidad en cuestión.
Dicho fenómeno de alteración, acaecido en el campo argentino, se debió al abandono de políticas proteccionistas y redistributivas, privatizaciones de las empresas de servicios, y desmantelamiento de institutos públicos de apoyo al agro. (Gras y Hernández, 2009: 13).

El espacio agrícola que queda, "...se ha transformado en un espacio sin agricultores, cuyos habitantes lo cultivan a tiempo parcial en ratos de ocio y en un medio muy deteriorado, que sufre el abandono de viejas normas organizativas"(EgeaFernández y Egea- Sánchez, 2008: 100).

Es imprescindible pensar una producción estable dentro del contexto de una organización social que proteja la integridad de los recursos naturales y que asegure la interacción armónica de los seres humanos, el agroecosistema y el medio ambiente. La respuesta se encuentra en el paradigma agroecológico; y con ello, aparece una nueva visión sobre el ambiente, y en consecuencia sobre nosotros mismos. Se plantea una mirada agroecológica fundada en principios ecológicos básicos para estudiar, diseñar y manejar agroecosistemas productivos y conservadores del recurso natural, culturalmente sensibles, socialmente justos y económicamente viables. Se alienta a conocer la sabiduría y habilidades de los campesinos e identificar el potencial sin límite de re-ensamblar la biodiversidad a fin de crear sinergismos útiles que doten a los agroecosistemas con la capacidad de mantenerse o volver a un estado innato de estabilidad natural. La sustentabilidad no es posible sin preservar la diversidad cultural (Altieri, 1999: 9-10)².

En este aquí y ahora, adquiere un papel vital la escuela rural en el tratamiento y cuidado del "patrimonio natural y cultural de valor biológico y sociocultural" (Egea-Fernández y EgeaSánchez, 2008: 101) que posee la zona. Dicha entidad, que suele ser muy pobre en términos de capital material; es muy rica en capital humano -conocimientos formales e informales-, capital cultural -normas y visiones del mundo, en constante evolución-, y en capital social -relaciones sociales de confianza y cooperación (Durston, 2002:92). Es en el medio rural donde queda mayormente de manifiesto, que los procesos educativos trascienden el espacio estrictamente escolar, que su calidad y efectividad mantienen una estrecha relación con los nexos entre docentes y padres de familia, entre contenidos escolares y preocupaciones, conocimientos e intereses de los contextos locales. La importancia y el valor de la escuela en el medio rural son indiscutibles, sobre todo por su posible efecto promotor en términos de cohesión y desarrollo sociocultural para cada comunidad en particular (Turcott y otros, 1998). 
3 Para resguardar la identidad de la institución escolar no se utiliza su nombre propio; en cambio se recurre a una sigla que se corresponde con las iniciales de su nombre.
A través de la práctica radiofónica y la problematización de temáticas ambientales y agroecológicas -intercambio de saberes- se promovió la construcción de la realidad por parte de niños y niñas sobre la promoción del cuidado del hábitat local; dicha producción de conocimiento quedó materializada en una experiencia radiofónica concreta.

El análisis de un caso particular -escuela MACP ${ }^{3}$ de Las Calles, provincia de Córdoba- permitió visibilizar alocuciones construidas por los niños en torno a la realidad socioambiental local; como así también prever el ejercicio activo de la población en el cuidado del ambiente.

La primera y segunda parte de esta presentación, introducen y contextualizan el estado de situación en el Valle de Traslasierra. En un tercer momento, se despliegan los diversos enfoques utilizados para estudiar la realidad; la realidad del hábitat local, que es interpretada y significada, un significado subjetivo que convierte a un mundo en coherente. De esta forma, comienza a encuadrarse el tema planteado. La cuarta parte, plantea la metodología de trabajo utilizada. El quinto momento, describe la creación de un espacio en la comunidad, donde los niños comunican sus intereses, gustos y opiniones; convirtiéndose de este modo en promotores y multiplicadores para la defensa y el resguardo del medio ambiente. La sexta, exhibe un breve cuadro de situación de los resultados obtenidos en los talleres de Comunicación y Ambiente, desplegados en el marco escolar.

\section{La construcción de la realidad socioambiental en el Valle de Traslasierra}

La comunidad de Las Calles, se caracteriza por la combinación de lo rural y lo turístico. El avance de formas de producción modernizadas, que incorporan lo transnacional al desarrollo regional, destradicionalizan y desarticulan los sistemas productivos campesinos históricos y las actividades de la población local. Dicha situación convoca a reflexionar sobre la construcción de un espacio donde la propia localidad pueda pensarse a sí misma, es decir, su realidad del mundo de la vida cotidiana en el aquí y ahora de su presente.

La elección de intervenir esta localidad en detrimento de otras, no fue aleatoria. Surgió a partir de acceder a una beca de extensión otorgada por la Universidad Nacional de Córdoba (2010). Dicho acontecer, facilitó el establecimiento del primer vínculo con informantes clave y el resto de entrevistados. Además, el desarrollo de este proyecto se debió a su articulación con un plan marco: "Aportes para la construcción de un desarrollo socio-territorial agroecológico en comunidades de Traslasierra de Córdoba". Desde hace 
dos años, un equipo interdisciplinario -conformado por ingenieros/as agrónomos, biólogos/as y comunicadores/as sociales- desarrolla diversas actividades en la zona. Así, se pensó que la recuperación de saberes ambientales y agroecológicos -a partir de un ciclo de programas radiales en la escuela primaria- sería una arista fundamental en la promoción de aportes para la construcción de un desarrollo socio-territorial agroecológico en comunidades rurales.

\section{Traslasierra, distintas perspectivas teóricas}

Para contextualizar sociológicamente este trabajo se cita a Peter Berger y Thomas Luckmannn, inscriptos en la perspectiva de la "sociología del conocimiento". Dicha postura es la base teórica fundamental. Por otro lado, se apela a los aportes de Paulo Freire, en la perspectiva de la "educación popular"; y Laura Vargas Vargas y Graciela Bustillos, quienes complementan el enfoque de la mano de "técnicas participativas". Se hace necesario enriquecer las conceptualizaciones con los aportes de otros autores. Todo esto, a fin de lograr una minuciosa mirada sobre la realidad intervenida. Así, se conceptualiza el lenguaje desde la óptica de Ludwig Wittgenstein. La recuperación de saberes se entiende en Boaventura De Sousa Santos, desde una "epistemología del sur" y el surgimiento de un conocimiento contra-hegemónico. Y el diálogo de saberes, como la técnica democrática para obtener dicho conocimiento. En tanto, la dimensión local y el enfoque agroecológico y ambiental, son definidos desde investigaciones contemporáneas: Miguel Altieri y José Egea-Fernández y José Egea- Sánchez. No centrarse en una única postura y nutrirse de conceptos varios ilustrados por la realidad-implicó la construcción de un marco teórico singular.

La comunicación radiofónica, un juego de niños y niñas. El trabajo aquí expuesto parte de una experiencia educativa que tiene como base el modelo de comunicación centrado en la construcción social de la realidad, parte de las condiciones sociales de producción de los discursos, tiene en cuenta las experiencias y saberes locales de sus interlocutores, y privilegia la producción colectiva de sentido (Gerbaldo, 2007: 14). En este escenario, la radio se concibe como un espacio de producción, de intercambio y encuentro, estableciéndose un vínculo entre los niños y el medio de comunicación, desde el juego y la creatividad.

La recuperación de saberes ambientales fue una puerta de ingreso al mundo del sentido común de los estudiantes primarios. "Las representaciones sociales son simultáneamente el producto y el proceso de una actividad mental por la cual 
un individuo o un grupo reconstituyen lo real con lo que está confrontado y le atribuye una significación específica" (Abric, 1994). En este sentido, la radio participativa ofreció a los niños la posibilidad de una relación en triple sentido: con los medios de comunicación; con el ambiente como parte de éste y como actor de los cambios que en él ocurran; y con la sociedad en un compromiso comunitario (aspectos no contemplados con austeridad en la educación formal). Además, permitió percibir, definir y plantear problemas a partir de la comprensión y racionalización de la situación socioambiental local. Para ello, se partió de un abordaje interdisciplinario, que reconoció la diversidad de concepciones culturales que conviven y confrontan en el territorio, ofreciendo su impronta de identidad y sentido de pertenencia, de valoración, rescate de conocimientos y saberes locales, de desigualdades e inequidades presentes, y de condiciones de los ecosistemas locales y regionales. Finalmente, para lograr la consolidación de la organización social, fue fundamental el enfoque de la investigación que procura la participación real de la población involucrada en el proceso de objetivación, análisis e investigación de la realidad en estudio (...) (Ryan y otros, 2009).

El enfoque agroecológico y la ecología de saberes. "La defensa de los recursos naturales y la revalorización de la 'agricultura tradicional' para que ésta sea un elemento de resistencia al proceso de marginación económica y degradación socioambiental de la sociedad rural, es una reivindicación presente en ciertos sectores de las sociedades rurales $(\ldots)$.

Este trabajo tuvo como base, colaborar en la localidad de Las Calles, y por extensión en el Valle de Traslasierra, para elaborar,-a largo plazo- propuestas de desarrollo rural endógeno desde un enfoque agroecológico. Se basó en imprimir a las acciones una praxis social, política y económica transformadora de la naturaleza de las relaciones sociales, y de la dinámica degradadora de la naturaleza y de la sociedad, impuesta por el modelo de desarrollo hegemónico. En tal sentido, fue fundamental otorgarle un papel central a la dimensión local como portadora de un potencial endógeno. Es desde la articulación del conocimiento campesino con el científico, que puede darse la implementación de sistemas de agricultura alternativa potenciadores de la biodiversidad ecológica (Altieri, 1983 y 1989; Caroll et al., 1990) y sociocultural (Sevilla Guzmán y otros, 1996: 42).

De Sousa Santos (2006) plantea, a partir de la "sociología de las ausencias", que existen diferentes modos de producir ausencias en la racionalidad occidental que las ciencias sociales comparten. Una de ellas es "la monocultura del saber 
y del rigor, la idea de que el único saber riguroso es el saber científico, y por lo tanto, otros conocimientos no tienen la validez ni el rigor del conocimiento científico. Esta monocultura reduce de inmediato, contrae el presente, porque elimina mucha realidad que queda afuera de las concepciones científicas de la sociedad, porque hay prácticas sociales que están basadas en conocimientos populares, conocimientos campesinos pero que no son evaluados como importantes o rigurosos..." "Al constituirse como monocultura (como la soja) destruye otros conocimientos (...) alternativos. Reduce realidad porque 'descredibiliza' no solamente a los conocimientos alternativos, sino también a los pueblos, los grupos sociales cuyas prácticas son construidas en esos conocimientos alternativos" (De Sousa Santos, 2006: 23-24). Por ejemplo, para los campesinos, la productividad de la tierra no es definida en un ciclo de producción sino en varios (la tierra es productiva un año y al año siguiente no se la cultiva para que descanse). Entonces, se está hablando de otra lógica productiva, una lógica propia de la racionalidad occidental. Es decir, cuando nacieron los productos químicos en la agricultura, la tierra paso a ser productiva en un ciclo de producción, porque los fertilizantes cambiaron el concepto de productividad de la naturaleza, que apareció al mismo tiempo que el concepto de productividad en el trabajo. Todo lo que no es productivo en este contexto es considerado improductivo o estéril. Aquí, la manera de producir ausencia es con la 'improductividad' (De Sousa Santos, 2006: 25). Y en este punto se enlaza la pugna agroecología - agricultura industrializada.

A partir de la "ecología de saberes" (...) se pretende que el saber científico dialogue con el saber popular, con el saber campesino.

En la misma línea, el diálogo de saberes, es la "expresión de una metodología democrática para construir en la diversidad, en la inclusión y en la participación; es también un método riguroso en su enfoque paradigmático, como contraposición al racionalismo científico característico de la sociedad contemporánea y aún presente en la sociedad moderna, que niega la existencia de métodos alternativos para la generación del conocimiento. Dialogar sobre los saberes tradicionales, es también innovar recuperando el conocimiento ancestral, que dista mucho de las prácticas obsoletas de la técnica y tecnologías modernas que no conciben al ciudadano común como autor del desarrollo y pretenden con sus libretos cientificistas convertirlo en sujetoobjeto del mismo" (Guzmán López, 2009). "(...) Los diálogos de saberes 0 entre saberes, buscan reconocer las distintas visiones, dando espacio a paralelismos y diferencias, útiles en 
la construcción de nuevo conocimiento" (...) (López y Hernández, 2009: 13). Se busca resaltar acciones que se den en el marco del mundo de la vida de los sujetos y frente al embate permanente del sistema dominante.

Otorgarle la palabra a culturales tradicionales, nativas y campesinas es una manera de generar resistencia frente a los discursos económicos del consumismo sin fronteras, de las ideologías individualistas y dominantes.

Realidad socioambiental en Traslasierra. El estado de situación -descripto párrafos arriba- en la comunidad de Las Calles, fue clave al momento de propiciar un acercamiento entre la escuela y la radio. Se constituyó un espacio de reflexión, construcción y participación con y desde los niños/as en torno a la conservación de un ambiente sano. Berger y Luckmannn (1968: 38-43) sustentan que la realidad se construye socialmente. "La realidad de la vida cotidiana es una construcción intersubjetiva, un mundo compartido, lo que presupone procesos de interacción y comunicación mediante los cuales comparto con los otros y experimento a los otros. Es una realidad que se expresa como mundo dado, naturalizado, por referirse a un mundo que es común a muchos hombres".

Las herramientas a las que se hace referencia se conceptualizan en términos del lenguaje; se trata del "sistema de signos más importante de la sociedad humana ya que la vida cotidiana, por sobre todo, es vida con el lenguaje que comparto con mis semejantes y por medio de él.

La trasmisión de conocimiento a través de la creación de distintos formatos radiales -boletines informativos, sociodrama, lectura de cuentos y reportajes- permitió interpelar a la comunidad en un mensaje intersubjetivo. Por lo tanto, la comprensión del lenguaje fue esencial para la comprensión de la realidad de la vida cotidiana. Por su parte, Ludwig Wittgenstein sostiene que no hay pensamiento sin lenguaje, sino pensamiento en el lenguaje; y que, a fin de cuentas, la experiencia es siempre pensada y sentida lingüísticamente. Conocemos el mundo en la medida, siempre de modo tentativo, que lo designamos con palabras y lo construimos sintácticamente en enunciados, es decir, a medida que y en la medida en que lo empalabramos (Chillón.1998: 68-70). La transmisión de conocimiento, a partir de la experiencia radiofónica, posibilitó un mayor encuentro entre la escuela y la comunidad para generar y aprehender una búsqueda conjunta de soluciones a problemas locales. "Un pensar crítico, a través del cual los hombres se descubren en situación" (Friere 2005: 136). Más allá de la percepción sensorial inmediata del entorno o del juego interior con las 
sensaciones registradas en la memoria, el mundo adquiere sentido solo en la medida en que lo traducimos lingüísticamente; de otro modo, solo sería para nosotros una barahúnda incoherente de sensaciones -táctiles, olfativas, visuales, acústicas, gustativas- suscitada por el entorno más inmediato aquí y ahora (Chillón, 1998: 70).

El desafío que se propusieron los talleres de Comunicación y Ambiente en la escuela de Las Calles fue: llenar de mensajes el espacio, tipificar la situación socio-ambiental de la zona, tomar la palabra; poner de manifiesto las prácticas discursivas de los niños en torno a la problemática socio-ambiental de la realidad local; y generar una interrelación e interacción entre los actores sociales de la comunidad.

\section{Estrategia metodológica}

El vínculo universidad-sociedad existe a partir del diálogo y el reconocimiento y valorización tanto de la igualdad como de la diferencia de saberes (científico humanístico y popularsocial). Las respuestas que la institución educativa brinda a las demandas sociales permiten la articulación de conocimientos. Los proyectos extensionistas ofrecen el contacto directo con la práctica a miembros universitarios, fortaleciendo espacios de sentido en diversas comunidades, donde estos son puestos en práctica. Se atiende a la reflexión de los participantes sobre su realidad cotidiana, los saberes que traen consigo del mundo exterior y la transferencia de estos conocimientos a diversas producciones concretas; siempre estimulando el aprendizaje socialmente significativo. La producción y reciprocidad de conocimientos permite adquirir nuevas herramientas y competencias para intervenir en la vida cotidiana desde el compromiso y el espíritu crítico. El diálogo potencia espacios de debate. Favorecer este encuentro -a partir de actividades en campo- es mutuamente enriquecedor: la localidad, grupo social, institución y en definitiva los destinatarios del proyecto mismo, obtienen aportes para la resolución de problemas específicos; y la universidad adquiere insumos para actualizar, repensar y redefinir los perfiles profesionales y áreas temáticas prioritarias sobre las cuales producir conocimiento. La interacción posiciona a la Universidad frente a una situación de escucha, reconociéndose también -a sí misma- con demandas hacia la sociedad.

Aquí, la extensión - entendida desde el diálogo de saberes- se pensó en términos de interdisciplinariedad y por tanto, desde una relación diádica: por un lado, la idea de establecer un diálogo entre diversas disciplinas fue pertinente para la construcción y abordaje de un objeto de estudio y trabajo. 
"La cooperación entre varias disciplinas o sectores heterogéneos de una misma ciencia lleva a interacciones reales, es decir, hacia una cierta reciprocidad de intercambios que dan como resultado un enriquecimiento mutuo" (Piaget citado por Longobardi, 1992 y Martínez de Aguirre, 1998). Y por otro lado, el intercambio de conocimientos entre el extensionista y los destinatarios del proyecto, fue pieza angular en la constitución, revalorización y reconocimiento del "otro". De Sousa Santos (2010) -desde una epistemología del sur- plantea "el reclamo de nuevos procesos de producción y de valoración de conocimientos válidos, científicos y no científicos, y de nuevas relaciones entre diferentes tipos de conocimiento (...)".

Así se delineó la existencia de dos horizontes disimiles, pero complementarios, para entender la intervención interdisciplinaria. La pretensión de dicha postura es contribuir a complejizar el concepto de extensión. No solo en el nivel de lo teórico, sino y básicamente desde una decisión política en la práctica, en la realidad de la vida cotidiana de la mediación. Son los actores quienes - a través del lenguaje y de sus prácticas- construyen el sentido de la interdisciplinariedad, que luego aplican en sus actividades cotidianas. Por ello, se convirtió en esencial entender dicho trabajo como una estrategia de intervención que organizó los pensamientos que actuaron sobre la realidad concreta para luego operar sobre ella. Se trata de una destreza de relacionamiento para la intervención profesional, que se ubica en el ámbito del quehacer cotidiano. Debe ser incorporada en términos reflexivos. No implica simplemente un trabajo conjunto y compartido. Su aspecto más importante radica en "la revaloración del otro, el reconocimiento de sus saberes, las necesidades mutuas, y la corresponsabilidad" (Fior de Leguizamón, 2008:48). Por este motivo se plantean dos niveles de interdisciplina; ya que es esencialmente en el orden del accionar común - y no el conceptual- donde se establecen encuentros, logros y creaciones conjuntas.

En este caso particular, y por la temática abordada, el trabajo se llevó adelante de la mano de conocimientos en agronomía y comunicación social. El carácter emergente de la propuesta, se debió a sus presupuestos políticos. La escuela se entiende como espacio esencial para la problematización ambiental y agroecológica; motivo convocante del proyecto extensionista. Trabajar desde el desarrollo rural agroecológico (...) propuso el abordaje de la realidad rural desde la perspectiva de la problemática social, en contraposición a las prácticas intervencionistas para imponer estilos de vida. Parafraseando a Amartya Sen, como la construcción de una "opción social" donde, se ponen en discusión objetivos y 
posibilidades de transformación económica, social, ambiental y política (Veiga, 2005). En tanto, desde la Educación Popular, se partió de las diferentes situaciones y problemas que enfrentan los sujetos en su vida, y que en un programa educativo se plantearon como temas a desarrollar. Desplegar un proceso de teorización sobre esa práctica -no como un salto a lo teórico sino como un proceso sistemático, ordenado, progresivo y al ritmo de los participantes- permitió ir descubriendo los elementos teóricos y profundizar de acuerdo al nivel de avance del grupo. De esta manera, lo cotidiano, lo inmediato, lo individual y parcial se ubica dentro de lo social, lo colectivo, lo histórico, lo estructural. El proceso de teorización permite regresar a la práctica para transformarla, mejorarla y resolverla; regresar con nuevos elementos que permitan que el conocimiento inicial, la situación, el sentir del cual partimos, ahora nos lo podemos explicar y entender, integral y científicamente. Por lo tanto, se puede fundamentar y asumir de manera consiente compromisos o tareas. Aquí es cuando se puede decir que la teoría se convierte en guía para una práctica transformadora.

Las "técnicas participativas" generan realmente un proceso de aprendizaje ya que permiten: desarrollar un trabajo colectivo de discusión y reflexión; como así también colectivizar el conocimiento individual, enriquecer éste y potenciar realmente lo grupal (Vargas Vargas y Bustillos, 1985). La Educación Popular se define por su "...concepción dialéctica, de clase, estrechamente ligada a la construcción de un conocimiento liberador (...). No puede reducirse a un simple aspecto de contenido y forma, sino que implica, necesariamente, adoptar una metodología que le de coherencia a ambos aspectos, pero siempre dentro de la definición señalada que la ubica ligada al quehacer del pueblo organizándose y a los intereses del cambio y la construcción de una nueva sociedad" (Vargas Vargas y Bustillos, 1985).

A partir del desarrollo de dos instancias: práctica radiofónica y problematización de temáticas ambientales y agroecológicas se alentó la participación protagónica de los niños y niñas en la gestión y realización de medios y mensajes; y la promoción de sus derechos.

La metodología defendida exigió, por esto mismo, que en el flujo de la investigación vayan haciendo ambos sujetos de la misma, tanto los investigadores-extensionistas- como los hombres del pueblo que, aparentemente, serían su objeto. (...) En el proceso de búsqueda de la temática significativa, ya debe estar presente la preocupación por la problematización de los propios temas. Por sus vinculaciones 
con otros..." (Freire, 2005: 132-134).

Entonces, con el propósito de recuperar saberes ambientales y agroecológicos a partir de la producción de un ciclo de programas radiales en la escuela, se utilizaron elementos de comunicación popular desde el planteo de dos módulos temáticos complementarios y sistemáticos: problematización socio-ambiental (contenido de los programas) y producción radiofónica (formato y lenguaje radiofónico). Se utilizó como herramienta la dinámica de taller, favoreciendo el encuentro, las experiencias, y las reflexiones conjuntas. Así, se generó un espacio participativo, en son de promover el interés por la temática socio-ambiental, la producción de nuevos aprendizajes; como así también fortalecer la identidad personal y colectiva. Se trabajó con 36 alumnos de $4^{\circ}, 5^{\circ}$ y $6^{\circ}$ grado (rango etario 9 a 12 años) ${ }^{2}$. La transmisión de conocimientos -desde los niños hacia el ámbito familiarpromovió el encuentro de la población en el cuidado del hábitat local. La elección del medio radial se consideró por su capacidad de inserción en el contexto próximo inmediato; además, por su vinculación a las prácticas sociales y el compromiso comunitario. El planteo inicial del trabajo se efectuó con reuniones de 2 horas cada 20 días, durante 6 meses. Esta situación fue revertida; así desde el inicio, los talleres se desarrollaron cada 15 días. Ya en el campo, y a partir de una reunión con el director de la institución, se consideró con certeza que la continuidad de los encuentros sería un factor clave para consolidar un verdadero ámbito de reflexión en la escuela rural. Además, en términos pedagógicos, fue fundamental dicho cambio para propiciar el desarrollo de las competencias de los niños y niñas. Con este mismo propósito, las grabaciones de los programas se llevaron adelante semanalmente.

\section{Los niños y niñas tienen la palabra}

Como productora de sentido, la comunicación es un espacio donde la cultura se visibiliza y reproduce. La estrecha vinculación entre el medio de comunicación radiofónico y la escuela, permitió responder a las condiciones espaciales: a través de orientar la conformación de un grupo con capacidad de coordinar las acciones de los actores y las instituciones territoriales involucradas en el proceso de desarrollo agroecológico; como así también fomentar la producción ecológica de alimentos (intercambio de saberes de técnicas productivas) y fortalecer los espacios 
interinstitucionales a través del asociativismo y el cooperativismo. Además, por ser el medio de mayor alcance en la zona, ofreció a la comunidad la posibilidad de incidir en las decisiones relativas a su propio desarrollo.

La construcción de la realidad ambiental -por parte de niños y niñas- a través de la herramienta radial admitió un vínculo lúdico y creativo.

El instrumento fundamental de trabajo -en la producción radiofónica- es la palabra; y por ello su importancia. Como seres simbólicos, los humanos tienden a actuar conforme a los significados que atribuyen a las representaciones que hacen acerca de la realidad. Es a través del lenguaje, como portador de significados convencionales, que se puede compartir la misma imagen mental acerca de un objeto o de un determinado estado de cosas. Esta importancia del lenguaje -como portador de significados y representacionesya fue advertida por el primer Wittgenstein (Arce Carrascoso, 1999:195-198) al sostener la conexión isomórfica entre lenguaje y mundo, evidenciando con ello que la función principal del lenguaje es figurar el mundo; y constituye una forma específica del proceso de construcción social de la realidad.

Se vive en el mundo del sentido común de la vida cotidiana equipado con cuerpos específicos de conocimiento. La legitimación produce nuevos significados que sirven para integrar los ya atribuidos a procesos institucionales dispares. No es solo cuestión de valores: siempre implica también conocimiento. No solo indica al individuo porqué debe realizar una acción y no otra; también le indica porque las cosas son lo que son.

Los niños elaboraron sus enunciados lingüísticos ambientales y agroecológicos (...) con el ingente acervo de saberes que heredaron por tradición. No es aceptable subestimar la importancia de la dialéctica incesante entre lo viejo y lo nuevo, pues sin ella es inconcebible cualquier nueva producción de sentido: el haz de ideas y creencias que forman la visión del mundo de cada niño-comunicador hinca sus raíces en el humus de la cultura, esto es, en la tradición heredada. En tal caso, la recuperación de saberes sobre el ambiente, la gestión y el uso de los recursos naturales y de los agroecosistemas, representa un elemento importante de la agrodiversidad. Este patrimonio inmaterial, transmitido de padres a hijos durante miles de años, no se encuentra en los libros de texto y su valor es incalculable. Sin embargo, al igual que los paisajes y los recursos genéticos locales para la alimentación, se encuentran en grave peligro de extinción. (...) Esto, sin entrar a valorar diferentes oficios en el medio rural desaparecidos en la actualidad. (...) La supresión de este 
patrimonio natural y cultural, de valor biológico, paisajístico y sociocultural incalculable no se puede permitir. Se han heredado del pasado muchos recursos que se deben mantener en el futuro. Esta falta de sensibilidad puede estar asociada al desconocimiento general de la sociedad del rico patrimonio ambiental y agrario que poseemos, de la problemática a la que está sometido, y del potencial que representan estos recursos endógenos para el mantenimiento de un mundo rural vivo y dinámico (Altieri y Nicholls, 2000). De esta manera, en el orden de los contenidos (programa radial), la construcción de la realidad generada por los estudiantes primarios, proveyó información sobre la manera de concebir su entorno social, cultural y ambiental. Entonces, se volvió primordial el diálogo con productores, docentes, familiares e instituciones de la comunidad.

"La comunicación en (y no para) la educación y la comunicación educativa dialógica, tienen sentido como construcción del sujeto histórico colectivo en lucha por la libertad" (Huergo, 2000: 19).

\section{Talleres de Comunicación y Ambiente}

Como herramienta y vehículo para la construcción de la realidad ambiental de la localidad de Las Calles, los niños y niñas llevaron adelante la producción de un programa radial. Dicha creación implicó una serie de pasos que los destinarios y protagonistas de este proyecto construyeron a través de un proceso de teorización sobre la práctica -no como un salto a lo teórico, sino como un proceso sistemático, ordenado, progresivo y al ritmo de los participantes- que permitió ir descubriendo los elementos conceptuales y profundizar de acuerdo al nivel de avance del grupo (Vargas Vargas y Bustillo, 1985). Sin duda, la familia, la escuela, y en este caso particular la radio, obraron como los mejores canales para vehiculizar dicha construcción.

Se comenzó desde la elección del tema y selección de los roles que cada uno iba a desempañar dentro del programa. A partir de la práctica problematizadora - dialógica, el contenido se organizó y constituyó en la visión del mundo de los educandos, encontrándose de este modo los temas generadores (Freire, 2005: 137). Dichos tópicos fueron: "cuidado de los animales"; "escases de agua"; "reciclado de la basura"; "deforestación"; "plantación de árboles"; "esquila de ovejas"; "cambio climático"; y "huertas y alimentos orgánicos". Para establecer esta síntesis, los alumnos previamente- efectuaron entrevistas y encuestas en el seno de la comunidad, como una manera de involucrase y empaparse a cerca de la situación contextual. Igualmente, la 
motivación al momento de investigar y sistematizar los conocimientos sobre ambiente y agroecología, fue alentadora. Mientras tanto, se incursionó en la musicalización y los efectos sonoros, a través de la creación artesanal de la artística radial, construyéndose de este modo la forma de auto denominación del programa. En las siguientes líneas se exponen algunas de las producciones:

Tema: ESCASES DE AGUA

Creación de artística: "¡Señor, señor, preste atención, que el derroche de agua está causando un problemón!"

Tema: CUIDADO DE LOS ANIMALES

Creación de artística: "¡Los animales están en peligro y los tienes que cuidar; y si hay un problema los tienes que ayudar!"

Tema: HUERTAS Y ALIMENTOS ORGÁNICOS

Artística: "¡Hay que cuidar la huerta para que nos de comida y que no de minerales para que nuestros cuerpo nos pida salud y vida!"

Tema: ESQUILA DE OVEJAS

Artística: "¡Nosotros vamos al campo para sacarle la lana a la oveja y después la tejemos!"

Los jingles presentados, son solo una muestra del trabajo generado por los alumnos de $4^{\circ}, 5^{\circ}$ y $6^{\circ}$ grado. Son sus primeros pasos en el ejercicio de la opinión y debate, en base a la resolución de problemas de la comunidad. Los saberes revelados se entienden, en términos de Boaventura de Sousa Santos. "La ecología de saberes comienza con la asunción de que todas las prácticas de relaciones entre los seres humanos, así como entre los seres humanos y la naturaleza, implican más de una forma de conocimiento, y por ello de ignorancia. Epistemológicamente, la moderna sociedad capitalista se caracteriza por favorecer prácticas en las que predomina el conocimiento científico". (...) Forjar la credibilidad para el conocimiento no científico, no supone desacreditar el científico. Simplemente implica su utilización contrahegemónica" (De Sousa Santos, 2010: 34).

Continuando con la elaboración del programa, definieron los géneros y formatos radiofónicos. La elección de una forma y no otra de manifestar la realidad implicó una determinada manera de construirla, representarla y transmitirla. Luego de profundizar en las particularidades de cada tipología existente, los niños -por grupo- eligieron la adecuada para acomodar la materia narrada: boletines informativos, sociodrama, reportajes, y creaciones de cuentos; toda una experiencia literaria. Para cada una de estas propuestas se elaboró el correspondiente guión radiofónico (pauta) y se 
organizó el contenido del programa.

La participación social infantil -aquí- tuvo como objeto el involucramiento en experiencias relacionadas con su entorno más cercano; espacio del que se dispone de información suficiente. El bagaje de conocimiento proveniente del mundo exterior, en complemento con las herramientas que incorporaron en los talleres, y su mirada sana y nítida, otorgó al conocimiento una validez sincera y sentida. Una noción de verdad, a la que muchos adultos no pueden acceder, ya sea por presiones externas o de coyuntura.

Con el fin de promover la apropiación del objetivo perseguido en la intervención - como sujetos activos, con presencia, voz y acción en sus comunidades- se prestó extrema atención al proceso de producción del conocimiento. En este sentido, fue significativo el fortalecimiento de la dicción, la expresión oral y por sobre todas las cosas, la participación y elaboración colectiva de conclusiones sobre temas abordados en clases. Al respecto, los niños, sostenían: "el cuidado del medio ambiente es el tema que les gustaría trabajar en los programa de radio, no solo por su interés personal, sino también porque dicha temática coincide con los intereses de los radioescuchas" (dato recabado a través de entrevistas realizadas a distintos integrantes de la familia). "Si nadie lo hace, nosotros tenemos que dar el primer paso para que la gente tome conciencia del daño que generamos al medio ambiente". Para desarrollar esta labor, ellos consideraron substancial disponer de acopio de conocimiento en la materia. Dicho acopio se incrementó paulatinamente en el aquí y ahora de su participación, a partir del diálogo con diferentes personajes de la comunidad y por medio de una exhaustiva investigación. Además, los pequeños consideraron fundamental para lograr su cometido - la toma de conciencia ambiental-: "utilizaremos como herramienta la radio".

En cada una de las actividades desplegadas quedó en evidencia desde un primer momento que los niños y niñas son conscientes del compromiso que les cabe como constructores de conocimiento. Así, se asumieron como seres responsables al hablar de dicha actitud como un valor moral, por su comunidad y su tierra. Precisamente, conscientes de ser formadores de opinión, decían: "La radio interpela a sus radioescuchas con sus propios gustos y los moviliza para reflexionar. Este tipo de prácticas promueve la participación y el enriquecimiento mutuo".

En todos los casos, los chicos advirtieron la falta de conciencia de la población sobre el cuidado del medio ambiente. Dicha desidia y negligencia comunal, es el motivo de mayor peso en el compromiso que asumen al embanderar esta causa. 
De este modo, la creación de un espacio en la comunidad y el ejercicio de la participación social niños y niñas-, fue fundamental para la consolidación de promotores y multiplicadores en la defensa y resguardo del medio ambiente.

Para finalizar, es importante resaltar una idea que transversaliza todo el andar del proyecto desarrollado en el Valle de Traslasierra. Los hombres no destruyen siempre la naturaleza; la transforman. Los cambios siempre inducen fenómenos de construcción y de destrucción. De esta manera, así como algunos grupos humanos están destruyendo el monte; "otros están haciendo florecer los desiertos, lo que significa en muchos casos ampliar la biomasa y aun la biodiversidad presente en esos ecosistemas" (Brailovsky, 2006: 17). Los dos casos representan la acción humana sobre la naturaleza. Si la solución es culpar al hombre, no hay nada que hacer, solo lamentarse. En este sentido, parafraseando a Brailovsky, planteamos la necesidad de una "aproximación diferente, una aproximación que comprometa a los integrantes de nuestras sociedades con el cuidado de la naturaleza. Dicha actitud supone un conocimiento específico de la problemática y del rol individual y social ante la misma. Es un compromiso social, acompañado por una actitud afectiva" (Brailovsky, 2006: 17).

\section{Referencias bibliográficas}

[1] ABRIC J.C. (1994): Pratiques sociales et representations. París. Presses Universitaires de France.

[2] ALTIERI, Miguel y NICHOLLS, Clara I. (2000): AGROECOLOGÍA. Teoría y práctica para una agricultura sustentable. la edición. México. Serie Textos Básicos para la Formación Ambiental.

[3] ARCE CARRASCOSO, José Luis. (1999): "Conocimiento y Comprensión: H. G. Gadamer". En: Teoría del Conocimiento. Sujeto, lenguaje, mundo. Madrid, Síntesis.

[4] BERGER, Peter L. y LUCKMANN, Thomas. (1968): La construcción social de la realidad. Buenos Aires. Amorrortu.

[5] BRAILOVSKY, Antonio E. (2006): Historia ecológica de Iberoamérica. De los Mayas al Quijote.la ed. Buenos Aires. Capital Intelectual.

[6] CHILLÓN, Lluís Albert. (1998): "El 'giro lingüístico' y su incidencia en el estudio de la comunicación periodística". En: Anàlisi. Quaderns de Comunicació i Cultura, núm. 22, Universidad Autónoma de Barcelona, Barcelona, Bellaterra.

[7] DE SOUSA SANTOS, Boaventura. (2010): Refundación del 
Estado en América Latina. Perspectivas desde una epistemología del sur. Lima. Programa Democracia y Transformación Global.

[8] DURSTON, John. (2002): "Gestión comunitaria de la escuela rural". En: El capital social campesino en la gestión del desarrollo rural. Díadas, equipos, puentes y escaleras. Santiago de Chile. Naciones Unidas. CEPAL.

[9] EGEA-FERNÁNDEZ, Jose $M^{a}$ y EGEA- SÁNCHEZ, Jose $M$. (2008): "Lugares de interés agroecológicos como espacio potencial para la producción ecológica". En: VIII CONGRESO SEAE sobre "Cambio climático, biodiversidad y desarrollo rural sostenible". IV Congreso Iberoamericano Agroecología y II Encuentro Internacional de Estudiantes de Agroecología y Afines. BULLAS (Murcia). España. SEAE - Sociedad Española de Agricultura Ecológica.

[10] FIOR DE LEGUIZAMÓN, Mabel B. (2008): Estrategias de Comunicación para la Articulación Investigación - Extensión. Vínculos y construcción de sentido en torno al concepto de interdisciplina en equipos de trabajo. Argentina. Asociación Cooperadora EEA INTA Oliveros.

[11] FREIRE, Paulo. (2005): Pedagogía del Oprimido. 2da ed. México. Siglo XXI editores.

[12] GERBALDO, Judith. (2007): Manual de Radio Participativa con niñas, niños y jóvenes Radioferoz! Córdoba. Ediciones CECOPAL.

[13] GRAS, Carla y HERNÁNDEZ Valeria. (2009): La Argentina rural. De la agricultura familiar a los agronegocios. Buenos Aires. Editorial Biblos Sociedad.

[14] GROSSO, Susana y ALBALADEJO, Christophe. (2009): "Los ingenieros agrónomos y la 'nueva agricultura': des/reterritorialización de la profesión". En: La Argentina rural. De la agricultura familiar a los agronegocios. Buenos Aires. Editorial Biblos Sociedad.

[15] GUZMAN LOPEZ, Samuel. (2009): Diálogos entre Saberes, Ciencias e Ideologías en Torno a lo Ambiental. Publiprint Ltda. Colombia.

[16] HERNÁNDEZ, Valeria. (2009): "La ruralidad globalizada y el paradigma de los agronegocios en las pampas gringas". En: La Argentina rural. De la agricultura familiar a los agronegocios. Buenos Aires. Editorial Biblos Sociedad.

[17] HUERGO, Jorge A. (2000): "Comunicación/Educación: itinerarios transversales". En: Comunicación-Educación. Coordenadas, abordajes y travesías. Colombia. Siglo del Hombre. 
[18] LONGOBARDI, R. (1992): Bases para un modelo interdisciplinario de capacitación gerencial. INTA. Dirección de Formación Superior y Desarrollo de Recursos Humanos. Documento $N^{\circ} 1$. Buenos Aires. Argentina.

[19] LÓPEZ CASTAÑO, Carlos E. y HERNÁNDEZ ARENAS, Uriel. (2009): Diálogos entre saberes, ciencias e ideologías en torno a lo ambiental. Colombia. Universidad Tecnológica de Pereira, Facultad de Ciencias Ambientales, Grupo Gestión de Cultura y Educación Ambiental.

[20] RYAN, Silvia y otros. (2009): CONSULTORIA: Asistencia Técnica para el Fortalecimiento Institucional para el Desarrollo Rural de la Provincia de Córdoba.

[21] SEVILLA GUZMÁN, Eduardo y otros. (1996): "La acción social colectiva en agroecología". En: Agricultura Ecológica y Desarrollo Rural. II Congreso de la Sociedad Española de agricultura Ecológica. Pamplona-Iruña. Córdoba. Instituto de Sociología y Estudios Campesinos, ETSIAM.

[22] TURCOTT, Verónica Rosana. (1998): Comunicación en la escuela y la comunidad: Cuaderno de autoestudio para el docente rural. Instituto Latinoamericano de la Comunicación Educativa.

[23] VARGAS VARGAS, Laura y BUSTILLOS, Graciela. (1985): Técnicas participativas para la educación popular. Tomo I. Buenos Aires. ALFORJA Publicaciones de Educación Popular. Editorial Humanitas.

[24] VEIGA, José ELi da. (2005): Desenvolvimento sustentable: o desafío do século XXI. Río de Janeiro. Ed. Gramond.

*Dda. Luciana-G. TRIMANO Adscripta. Investigadora. Escuela de Ciencias de la Información (ECI). Universidad Nacional de Córdoba (UNC) - Argentina. lucianatrimano@gmail.com

*Dra. Paulina-B. EMANUELLI Profesora Titular Taller de Metodología de la Investigación Aplicada. Investigadora. Escuela de Ciencias de la Información (ECI). Universidad Nacional de Córdoba (UNC) - Argentina. pemanuelli@hotmail.com

Fecha de recepción: 22/05/2012 Fecha de revisión: 01/09/2012

Fecha de preprint: 07/01/2013 Fecha de publicación final: 30/06/2013 